\title{
Çoklu-GNSS çözümlerinin performansının internet tabanlı Trimble RTX servisi ile incelenmesi
}

\author{
Gizem Sezer ${ }^{1}$ (D), Ali Hasan Doğan ${ }^{1}\left(\mathbb{D}\right.$, Bahattin Erdoğan ${ }^{1+(\mathbb{B})}$ \\ ${ }^{1}$ Yıldız Teknik Üniversitesi, Davutpaşa Kampüsü, Inşaat Fakültesi, Harita Mühendisliği Bölümü, Esenler, İstanbul, Türkiye.
}

Öz: Günümüzde jeodezik amaçlı yapılan çalışmaların birçoğunda Küresel Konumlama ve Uydu Sistemleri'ne (Global Navigation Satellite System, GNSS) ait veriler kullanılmaktadır. GNSS yapısında, GPS, GLONASS, Galileo ve BeiDou uydu sistemleri beraber değerlendirilerek konum bilgisi elde edilebilmektedir. Uluslararası GNSS Servisi (International GNSS Service, IGS) bu amaç doğrultusunda farkl sinyal ve uydu sistem verilerinin toplanmast, izlenmesi ve analiz edilmesi için Multi-GNSS Experiment (MGEX) adında bir proje başlatmıştır. Çoklu-GNSS çözümlerinin kullanılmaya başlamasından dolayl, bu çözümlerden elde edilen konum doğruluğunun belirlenmesi kullanıcılar açısından büyük önem arz etmektedir. Bu nedenle bu çalışmada; 10 noktaya ait GNSS verileri; GPS, GPS/GLONASS, GPS/GLONASS/Galileo ve GPS/GLONASS/Galileo/BeiDou olmak üzere 4 farklı senaryo ile değerlendirilmiş ve doğruluk analizleri gerçekleştirilmiştir. GNSS verilerinin değerlendirilmesi bir internet tabanl yazılım olan ve Çoklu-GNSS hassas mutlak nokta konumlama (Precise Point Positioning, PPP) çözüm kapasitesine sahip Trimble RTX servisi ile gerçekleştirilmiştir. Sonuçlar, sadece GPS çözümleri yerine farklı uydu kombinasyonlarını birlikte kullanarak daha yüksek doğrulukla konum bilgisi elde edilebileceğini göstermiştir. Ayrıca, Çoklu-GNSS çözüm doğruluklarının gözlem süresi ve enlem bilgisine bağlı olup olmadığ da incelenmiştir. Sonuçlara göre Çoklu-GNSS çözüm doğruluklarının sadece gözlem süresine bağlı olarak değiştiği vurgulanmıştır.

Anahtar Sözcükler: Çoklu-GNSS, Doğruluk, Hassas mutlak nokta konum belirleme, Trimble RTX servisi

\section{Investigation of the performance of the Multi-GNSS analysis with web based Trimble RTX service}

Abstract: Nowadays, Global Navigation Satellite Systems (GNSS) data are used in most of the geodetic studies. Positioning information can be obtained by using GPS, GLONASS, Galileo and BeiDou in the structure of the GNSS. International GNSS Service (IGS) has initiated a project, called Multi-GNSS Experiment (MGEX), to collect, track and analyze different signals and satellite system data. Since, the MultiGNSS solutions have started to use, positioning accuracy obtained from Multi-GNSS solutions is very important for users. Therefore, in this study, GNSS data of 10 stations were analyzed in 4 different scenarios as GPS, GPS/GLONASS, GPS/GLONASS/Galileo and GPS/GLONASS/Galileo/BeiDou. The GNSS data were processed using Trimble RTX service which is a web based precise point positioning $(P P P)$ software that is capable of processing Multi-GNSS data. Results have demonstrated that, it has been shown that the higher positioning accuracy can be obtained using different satellite systems together, instead of using GPS-Only. Moreover, accuracy of MultiGNSS solutions was investigated whether it depends on session duration and latitude or not. According to the results, it was emphasized that accuracy of Multi-GNSS solutions only depends on session duration.

Keywords: Multi-GNSS, Accuracy, Precise point positioning, Trimble RTX service 


\section{Giriş}

Küresel Konumlama ve Uydu Sistemleri (Global Navigation Satellite System, GNSS); mühendislik, navigasyon, meteorolojik çalışmalar gibi birçok alanda tercih edilmektedir. GNSS yönteminin klasik yöntemlere göre tercih edilme sebepleri; noktaların birbirlerini görme zorunluluğunun olmaması, günün her saatinde ve her türlü hava koşulunda çalışabilir olması şeklinde sıralanabilir. Her ne kadar GNSS yöntemi ile geçmişte yüksek doğrulukta konum bilgisi elde edilebilmesi için en az iki GNSS alıcısı (bağıl konum belirleme) ihtiyacı olsa da; son yıllarda geliştirilen hassas mutlak nokta konumlama (Precise Point Positioning, PPP) tekniği ile tek alıcı ile cm mertebesinde konum bilgisi elde edilebilmektedir (Gao \& Shen, 2001; Kouba \& Héroux, 2001; Zumberge, Heflin, Jefferson, Watkins, \& Webb, 1997).

PPP tekniği ilk olarak Zumberge vd. (1997) tarafından öne sürülmüş, daha sonra Kouba ve Héroux (2001) ve Gao ve Shen (2001) tarafından geliştirilmiştir. PPP tekniği her ne kadar mühendislik uygulamalarında maliyeti düşürse de, cm mertebesinde doğruluğa ulaşabilmesi için uzun yakınsama süresi gerektirmektedir (Geng, Teferle, Meng, \& Dodson, 2011). GNSS ile konum belirlemede genelde GPS uydu verileri tercih edilmektedir. Ancak, GPS uydularına farklı uydu sistemlerinin (GLONASS, Galileo, BeiDou) eklenmesi ile yakınsama süresi kısalabilmektedir (Bisnath \& Gao 2009; Rizos, Janssen, Roberts, \& Grinter, 2012; Toluc, 2016).

Uluslararası GNSS Servisi (International GNSS Service, IGS) farklı sinyal ve uydu sistem verilerinin toplanması, izlenmesi ve analiz edilmesi için Multi-GNSS Experiment (MGEX) projesini başlatmıştır. Bu projenin amaçlarından biri de farklı uydu sistemlerinin bir arada (Çoklu-GNSS) tüm özellikleri ile kullanılabilmesi olarak ifade edilmektedir (Montenbruck vd., 2017; URL-1). Bu bağlamda bilim dünyasında birçok çalışma gerçekleştirilmiştir. Li vd. (2015) Çoklu-GNSS çözümleri ile yakınsama süresinin \%70 kısaldığını, konum belirleme doğruluğunun ise \%25 arttığını vurgulamıştır. Xia vd. (2019) Galileo sisteminin diğer uydu sistemlerine olan katkısını incelemiştir. Çalışmada, Galileo kullanımının sadece GPS çözümlerine göre yakınsama süresini \%30.45, GPS/GLONASS kombinasyon süresini ise \%7.80 azalttığı gösterilmiştir. Ayrıca; Galileo’nun kinematik çözümlerde sadece GPS çözümlerinden elde edilen konum doğruluğunu \%20-30 oranında arttırdığ1 ifade edilmiştir. Wang vd. (2019) ise Çoklu-GNSS çözümlerinin yoğuşabilir su buharı miktar doğruluğunu \%10 arttırdığını göstermiştir. Öğütcü (2019)'da, 6 IGS istasyonunun farklı gözlem sürelerine ait Çoklu-GNSS verileri GipsyX yazılımı kullanılarak değerlendirilmiştir. Farklı uydu sistemlerinin özellikle kısa süreli gözlemlere büyük oranda katkı yaptığ1 belirtilmiştir. Öğütcü (2020) ise Galileo sisteminin yakınsama süresini statik çözümlerde düşey bileşende \%45, yatay bileşende $\% 50$; kinematik çözümlerde düşey bileşende $\% 62$, yatay bileşende $\% 89$ oranında kısalttı̆̆ını ifade etmiştir.

GNSS verileri akademik, ticari ve internet tabanlı yazılımlar kullanılarak değerlendirilebilmektedir. Öcalan, Erdoğan ve Tunalığlu (2013) internet tabanlı yazılımlar ile mm mertebesinde yatay konum doğruluğu elde edilebileceğini göstermiştir. Ayrıca, Albayrak, Erdoğan ve Erkaya (2020) ise internet tabanlı yazılımların jeodezik çalışmalarda kullanılabilir olduğunu ve gelecekte daha verimli olacağını vurgulamıştır. Literatür incelendiğinde ise uzun süreli gerçekleştirilen gözlemler dikkate alınarak hesaplanan güvenilir kestirim değerlerine göre GNSS çözüm doğruluklarının karesel ortalama hataları (Root Mean Square, RMS) ile incelendiği görülebilmektedir (Eckl, Snay, Soler, Cline, \& Mader, 2001; Soler, Michalak, Weston, Snay, \& Foote, 2006; Soycan \& Öcalan, 2011; Tut, Şanlı, Erdoğan, \& Hekimoğlu, 2013). Bu çalışmada, Çoklu-GNSS çözüm doğruluklarının internet tabanlı bir servis kullanılarak belirlenmesi amaçlanmıştır. Bu kapsamda, 10 adet noktaya ait 2, 4, 6, 8, 12 ve 24 saatlik Çoklu-GNSS verileri internet tabanlı Trimble RTX servisi kullanılarak analiz edilmiştir. Konum doğrulukları GPS (G), GPS/GLONASS (GR), GPS/GLONASS/Galileo (GRE) ve GPS/GLONASS/Galileo/BeiDou (GREC) olmak üzere dört farklı senaryo üzerinden incelenmiştir. Ayrıca, hem gözlem süresinin hem de nokta enlem bilgisinin ÇokluGNSS çözüm doğruluğuna etkisini irdelemek amacıyla doğruluk fonksiyonları belirlenmiştir. 


\section{Hassas Mutlak Nokta Konumlama}

PPP tekniği, tek bir GNSS alıcısı ile cm mertebesinde konum doğruluğu elde edilmesini sağlamaktadır. PPP tekniğinde, kod ve faz ölçülerinin iyonosferden bağımsız lineer kombinasyonları birlikte kullanılarak yüksek doğrulukta konum bilgisi elde edilmesi sağlanır (Gao \& Shen, 2001; Kouba \& Héroux, 2001). Bu sayede GNSS'in en büyük hata kaynaklarından biri olan iyonosfer hatası büyük oranda elimine edilmiş olur (Öcalan, 2015). Ancak, PPP tekniğinde mutlak konumlama ile tek alıcı kullanarak konum bilgisi elde edildiğinden, bağıl konum belirlemede olduğu gibi fark alma teknikleri ile diğer hata kaynakları giderilememektedir. $\mathrm{Bu}$ hata kaynaklarının farklı kurum ve kuruluşlar tarafından üretilen ürünler yardımıyla veya modellenerek giderilmesi gerekmektedir. PPP tekniğinin hata kaynakları Tablo 1'de özetlenmiştir.

Tablo 1: PPP hata kaynakları (Rizos vd., 2012)

\begin{tabular}{cl}
\hline Hata Kaynakları ve Modelleri & Düzeltme Türü \\
\hline \multirow{3}{*}{ Uydu Kaynaklı Hatalar } & Hassas uydu saat düzeltmeleri \\
& Uydu anteni faz merkezi offset değerleri \\
& Uydu anteni faz merkezi kayılıkları \\
& Hassas uydu yörüngeleri \\
& Diferansiyel grup gecikmeleri \\
& Görelilik koşulu \\
& Uydu anteni faz dönmesi \\
\hline \multirow{2}{*}{ Alıcı Kaynaklı Hatalar } & Alıcı anteni faz merkezi offset değerleri \\
& Alıcı anteni faz merkezi kayıllıları \\
& Alıcı anteni faz dönmesi \\
\hline \multirow{2}{*}{ Jeofiziksel Modeller } & Katı yeryuvarı gelgiti \\
& Okyanus yüklemesi \\
& Kutup gelgiti \\
& Plaka tektonik hareketleri \\
\hline \multirow{2}{*}{ Atmosferik Modelleme } & Troposferik gecikme \\
& Iyonosferik gecikme \\
\hline \multirow{2}{*}{}
\end{tabular}

$\mathrm{Bu}$ çalışmada veri analizleri internet tabanlı Çoklu-GNSS PPP servisi olan Trimble RTX kullanılarak gerçekleştirilmiştir (URL-2). Trimble RTX Eşitlik 1 ve 2’yi temel alarak çözümleri gerçekleştirmektedir (Doucet vd., 2012).

$P_{i, k}^{j}=\rho_{i}^{j}+c \Delta t_{i}-c \Delta t^{j}+T_{\dot{\mathrm{I}}}^{j}+I_{P, i, k}^{j}+b_{P, i, k}-b_{P, k}^{j}+m_{P, i, k}^{j}+\epsilon_{P, i, k}^{j}$

$\phi_{i, k}^{j}=\rho_{i}^{j}+c \Delta t_{i}-c \Delta t^{j}+T_{\dot{\mathrm{I}}}^{j}+I_{\phi, i, k}^{j}+b_{\phi, i, k}-b_{\phi, k}^{j}+\lambda_{k} N_{i, k}^{j}+m_{\phi, i, k}^{j}+\epsilon_{\phi, i, k}^{j}$

Burada; $P_{i, k}^{j}$ ve $\phi_{i, k}^{j}$ kod ve faz ölçülerini, $\rho_{i}^{j}$ uydu ve alıcı arasındaki geometrik uzaklığı, $c$ ışık hızını $\Delta t_{i}$ ve $\Delta t^{j}$ sırasıyla alıcı ve uydu saat hatalarını, $T_{\dot{\mathrm{I}}}^{j}$ troposferik gecikmeyi, $I_{P, i, k}^{j}$ ve $I_{\phi, i, k}^{j}\left(=-I_{P, i, k}^{j}\right)$ kod ve faz ölçüleri için iyonosferik gecikmeyi, $b_{P, i, k}$ ve $b_{\phi, i, k}$ alıcı kod ve alıcı faz hatalarını, $b_{P, k}^{j}$ ve $b_{\phi, k}^{j}$ uydu kod ve uydu faz hatalarını, $\lambda_{k} k$ frekansı için taşıyıcı dalga boyunu, $N_{i, k}^{j}$ başlangıç tamsayı faz belirsizliğini, $m_{P, i, k}^{j}$ ve $m_{\phi, i, k}^{j}$ kod ve faz ölçüleri için çok yolluluk etkisini, $\epsilon_{P, i, k}^{j}$ ve $\epsilon_{\phi, i, k}^{j}$ kod ve faz ölçüleri için gürültüleri ifade etmektedir.

Analizlerde Trimble-RTX tarafından üretilen hassas yörünge ve saat bilgileri kullanılmaktadır. Bu ürünlerin 1 saniyelik zamansal çözünürlüğe sahip olduğu belirtilmektedir. Yakınsama süresi yaklaşık 15 dakika olan Trimble RTX servisi ile statik çözümlerde mm mertebesinde doğrulukla konum bilgisi elde edilebilmektedir (Doucet vd., 2012). İyonosferik etki, iyonosferden bağımsız sinyal kombinasyonları ile giderilmektedir. Alıcı saat hataları, troposferin ıslak bileşeni ve gradyentler bilinmeyen olarak kestirilmektedir. Ayrıca, Tablo 1'de yer alan hata kaynakları Trimble RTX servisi ile çözümlerde göz önünde bulundurulmaktadır. 


\section{Veri Analizi}

Bu çalışmada IGS ağına ait 10 noktanın, 7-16 Haziran 2018 günlerine ait 10 günlük verisi değerlendirilmiştir (Şekil-1). Hesaplamalarda bu tarih aralıkları seçilirken; iyonosferik etkilerin ve manyetik fırtınaların etkisini en aza indirmek için Kpindeks değerleri de göz önüne alınmıştır (Şekil-2). Kp-indeks değeri küresel ölçekte jeomanyetik etkiyi ifade etmektedir ve 3 saatlik gözlemler sonucunda elde edilmektedir. Bu değer 0 ile 9 arasında değişmektedir. Bu çalışmada sınır değer “4” olarak belirlenmiştir. Literatürdeki çalışmalar, 4'ün üzerindeki değerlerin şiddetli jeomanyetik etkiyi ifade ettiğini belirtmiştir (Yapıc1, 2007; URL-3). Ayrıca, noktalar seçilirken Çoklu-GNSS çözümlerinin farklı enlemlerdeki etkileri de incelenmek istendiğinden nokta enlem bilgileri de dikkate alınmıştır. Tablo 2, yaklaşık nokta konum bilgilerini ve noktaların hangi günlerde hangi uydu sistemi verilerini içerdiğini göstermektedir.

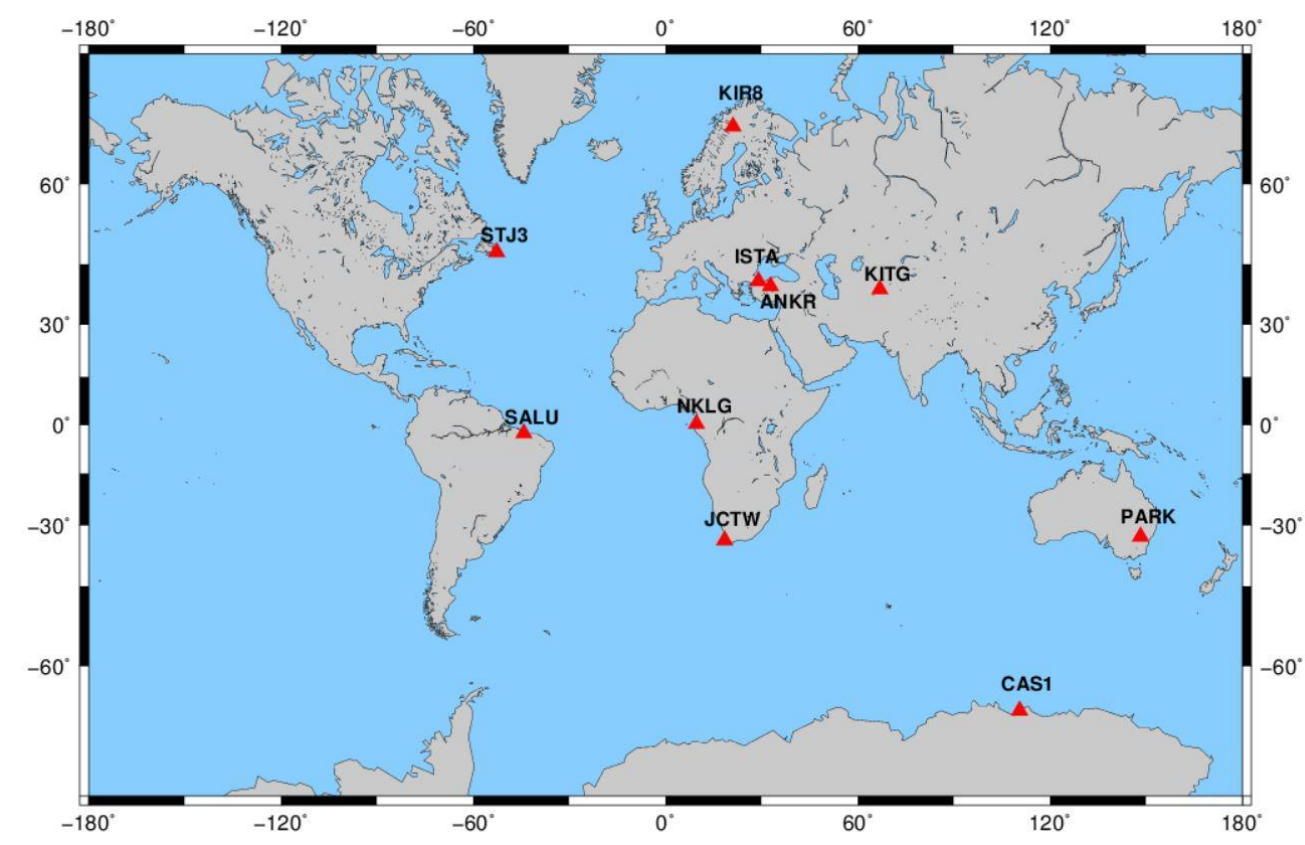

Şekil 1: Çalışmada kullanılan noktalar

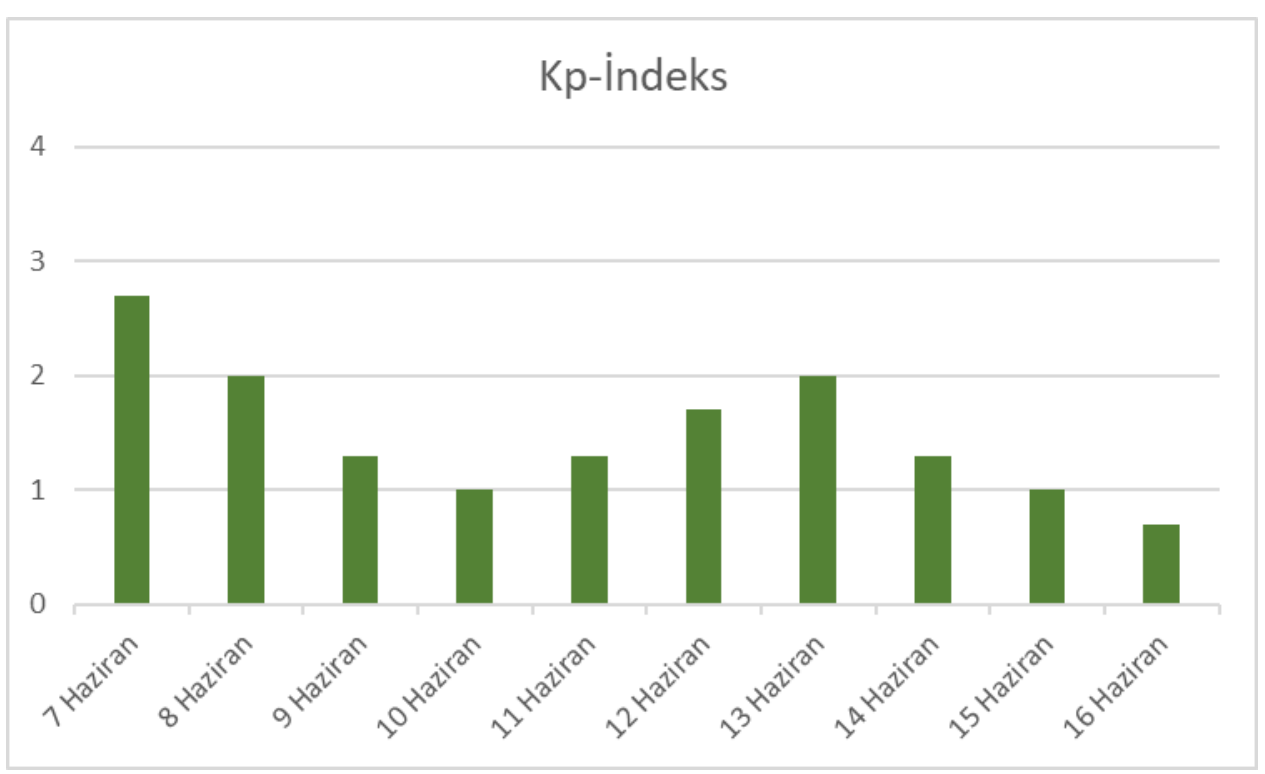

Şekil 2: Seçilen günlere ait Kp-Indeks değerleri (URL-3) 
Tablo 2: Çalışmada kullanılan nokta bilgileri (WGS84 elipsoidine göre)

\begin{tabular}{|c|c|c|c|c|c|}
\hline Nokta & Enlem $\left(^{\circ}\right)$ & Boylam $\left({ }^{\circ}\right)$ & Yükseklik (m) & DOY & Uydu Sistemi \\
\hline ANKR & 39.88737 & 32.75847 & 976.008 & $160-167$ & G-R-E-C \\
\hline CAS1 & -66.28336 & 110.51971 & 22.478 & $158-167$ & G-R-E-C \\
\hline ISTA & 41.10445 & 29.01934 & 147.240 & $158-167$ & G-R-E-C \\
\hline JCTW & -33.95143 & 18.46855 & 83.601 & $158-167$ & G-R-E-C \\
\hline KIR8 & 67.87755 & 21.06019 & 498.162 & $158-167$ & G-R-E-C \\
\hline KITG & 39.13339 & 66.88674 & 620.654 & $158-167$ & G-R-E-C \\
\hline NKLG & 0.35391 & 9.67213 & 31.498 & $158-167$ & G-R-E-C \\
\hline PARK & -32.99876 & 148.26461 & 397.374 & $158-167$ & G-R-E-C \\
\hline SALU & -2.59346 & -44.21248 & 18.977 & $158-167$ & G-R-E-C \\
\hline STJ3 & 47.59544 & -52.67831 & 154.516 & $158-167$ & G-R-E-C \\
\hline
\end{tabular}

DOY: Yılın günü (Day of Year), G: GPS, R: GLONASS, E: Galileo, C: BeiDou

Çoklu-GNSS çözümlerinden elde edilen konum doğrulukları GPS (G), GPS/GLONASS (GR), GPS/GLONASS/Galileo (GRE) ve GPS/GLONASS/Galileo/BeiDou (GREC) olmak üzere dört farklı senaryo üzerinden incelenmiştir. BeiDou uydu sisteminde küresel boyutta bazı bölgelerde uydu görünürlüklerinde BDS-2 uyduları kullanıldığında sorun yaşanmaktadır. Bu çalışmada da sadece BDS-2 uyduları kullanıldığından farklı gözlem süreleri için noktalarda görülen ortalama BDS-2 uydu sayılarını gösteren grafik Şekil 3’te verilmektedir. Literatürdeki çalışmalar incelendiğinde konum doğruluklarının ve güvenirliklerinin tekrarlılıklardan elde edilen RMS değerleri ile ilişkilendirildiği görülebilmektedir (Eckl vd., 2001; Erdoğan, Kayacık, \& Doğan, 2019; Erdoğan \& Doğan, 2019; Soler vd., 2006; Soycan \& Ocalan, 2011; Tut vd., 2013). Ayrıca GPS ile elde edilen konum doğruluklarının gözlem süresine bağlı olduğu birçok çalışmada gösterilmiştir (Eckl vd., 2001; Erdoğan \& Doğan, 2019; Şanlı \& Engin, 2009; Tut vd., 2013). Bu nedenle Çoklu-GNSS çözümlerinin gözlem süresine bağlı olup olmadığını belirlemek için veriler 2, 4, 6, 8, 12 ve 24 saatlik gözlem sürelerine bölünmüştür.

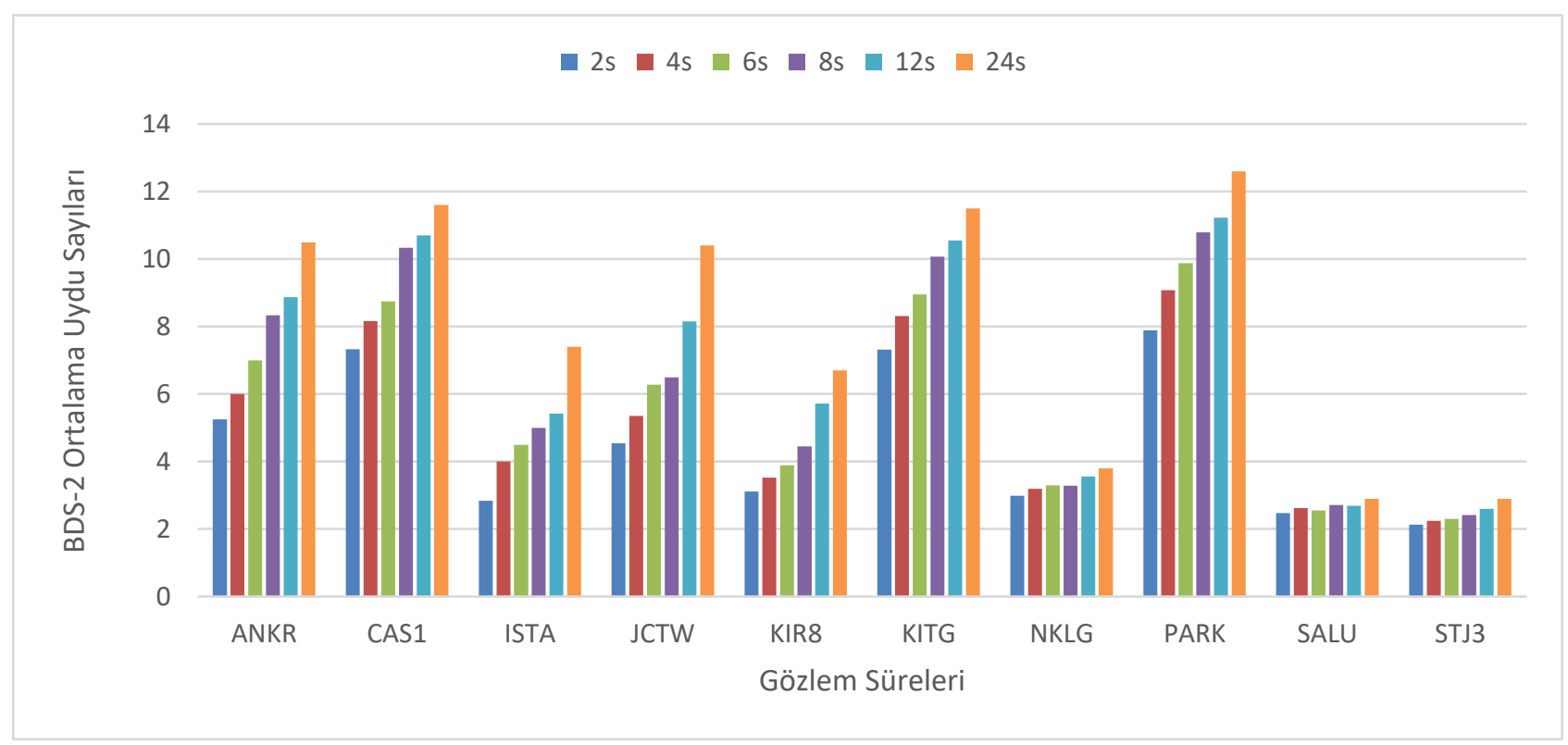

Şekil 3: Noktalardaki gözlem sürelerine göre ortalama BDS-2 uydu sayıları

Trimble RTX servisi kullanıcıya farklı datumlarda çözüm yapma imkânı tanımaktadır. Bu çalışmada çözümler ITRF2014 datumunda gerçekleştirilmiştir. Yazılım sonuç ürünü, ölçü epoğunda yer merkezcil Kartezyen koordinat sistemi koordinatlarını ve koordinatların varyans kovaryans bilgilerini içermektedir. Ancak bu çalışmada konum doğrulukları doğu 
(d), kuzey (k) ve yukarı (y) bileşenleri şeklinde incelenmek istendiğinden; yer merkezcil koordinatlar toposentrik koordinatlara Eşitlik 3 yardımıyla dönüştürülmüştür.

$\mathbf{R}=\left|\begin{array}{ccc}-\sin (\lambda) & \cos (\lambda) & 0 \\ -\sin (\varphi) \cos (\lambda) & \sin (\varphi) \sin (\lambda) & \cos (\varphi) \\ \cos (\varphi) \cos (\lambda) & \cos (\varphi) \sin (\lambda) & \sin (\varphi)\end{array}\right|$

$\left|\begin{array}{l}d \\ k \\ y\end{array}\right|=\mathbf{R} \times\left|\begin{array}{c}X-X_{R} \\ Y-Y_{R} \\ Z-Z_{R}\end{array}\right|$

Burada; $\mathbf{R}$, dönüşüm matrisini, $X, Y$ ve $Z$ yer merkezcil Kartezyen koordinatları, $X_{R}, Y_{R}$ ve $Z_{R}$ referans yer merkezcil Kartezyen koordinatları ve $d, k$ ve $y$ toposentrik koordinatları ifade edilmektedir. Referans koordinatlar her bir senaryo için 24 saatlik çözümlerin ortalaması olarak seçilmiştir.

$\mathrm{Bu}$ çalışmada Çoklu-GNSS çözümlerinden elde edilen konum doğrulukları; literatürdeki çalışmalarda olduğu gibi RMS değerleri ile ilişkilendirilmiştir. RMS değerleri hesaplanmadan önce koordinat serilerindeki uyuşumsuz ölçüler; \%50 kırılma noktasıyla en güvenilir Robust yöntemlerden biri olan Medyan yöntemiyle analizlerden çıkarılmıştır (Hampel, Ronchetti, Rousseeuw, \& Stahel, 2011; Hekimoğlu, 2005). Medyan yöntemi Eşitlik 4 ile ifade edilir.

$\operatorname{mad}=\left\{\begin{array}{rr}1.2533 \times \frac{1}{n} \times \Sigma \mid \mathbf{X}-\text { medyan }(\mathbf{X}) \mid, & \text { medyan }(\mathbf{X}-\text { medyan }(\mathbf{X}))=0 \\ 1.4826 \times \text { medyan }(\mathbf{X}-\text { medyan }(\mathbf{X})), & \text { medyan }(\mathbf{X}-\text { medyan }(\mathbf{X})) \neq 0\end{array}\right.$

Burada; X; kuzey, doğu ve yukarı bileşenleri için koordinat serilerini, $n$ ölçü sayısını ifade etmektedir. Bu yöntemde $\mid \mathbf{X}-$ medyan $(\mathbf{X}) \mid$ düzeltme değerleri $3 \times$ mad sınır değeri ile karşılaştırılır. Sınır değerden büyük olan düzeltmeye ilişkin koordinat değeri, koordinat serisinden çıkarılır. Uyuşumsuz ölçü analizinden sonra RMS değerinin hesabında Eşitlik 5 kullanılmıştır.

$\mathrm{RMS}=\sqrt{\frac{\sum_{i=1}^{n}\left(X_{i}-X_{R}\right)^{2}}{n}}$

Burada; $X_{i}$ i. koordinat değerini, $X_{R}$ referans koordinatı, $n$ ölçü sayısını ifade etmektedir.

Tablo 3’te tüm gözlem süreleri ve tüm senaryolar için Medyan yöntemi sonrası hesaplanmış RMS değerleri gösterilmektedir. RMS değerleri incelendiğinde, literatürdeki çalışmalarda olduğu gibi gözlem süresi arttıkça doğruluğun arttığı görülmektedir. Tablo 3 senaryolar özelinde incelendiğinde, 24 saatlik çözümlerin dışındaki tüm çözümlerde Galileo ve BeiDou sistemlerinin GPS ve GLONASS sistemlerine katkı sağladığı açıkça görülmektedir. Özellikle kısa süreli gözlemlerde Çoklu-GNSS çözümleri nokta konum doğruluğunu olumlu etkilemektedir. 24 saatlik çözümlerde GPS çözümleri ile Çoklu-GNSS çözümleri benzerlik göstermektedir.

Koordinat bileşenleri ayrı ayrı incelendiğinde yine benzer sonuçların elde edildiği görülebilmektedir. Doğu bileşeninde; 8 saatten daha kısa gözlem süresine sahip çözümlerde en yüksek doğruluk GREC kombinasyonu ile elde edilirken, 8 ve 12 saatlik gözlem sürelerinde GRE kombinasyonu en yüksek doğruluğu sağlamıştır. 24 saatlik çözümlerde ise sadece GPS çözümleri daha yüksek doğruluğa ulaşmıştır. Kuzey bileşeninde; 12 saatten daha kısa olan gözlem sürelerinde GRE ve GREC kombinasyonları ile daha yüksek doğruluk elde edilmişken; 12 ve 24 saatlik gözlem sürelerinde G ve GR kombinasyonları daha yüksek doğruluk sağlamıştır. Yukarı bileşeni incelendiğinde kısa süreli gözlemlerde Galileo ve BeiDou sistemlerinin 
GPS ve GLONASS sistemlerine büyük katk1 yaptığı görülmektedir. Bu sonuçlar Öğütçü (2019) ile de benzerlik göstermektedir.

Tablo 3.1: G ve GR senaryolarına ait farklı gözlem süreleri için RMS değerleri (mm)

\begin{tabular}{|c|c|c|c|c|c|c|c|c|c|c|c|c|c|}
\hline \multicolumn{2}{|c|}{ Senaryo } & \multicolumn{6}{|c|}{ G } & \multicolumn{6}{|c|}{ GR } \\
\hline \multirow{2}{*}{ Nokta } & \multirow{2}{*}{ Bileșen } & \multicolumn{6}{|c|}{ Gözlem Süresi (sa) } & \multicolumn{6}{|c|}{ Gözlem Süresi (sa) } \\
\hline & & 2 & 4 & 6 & 8 & 12 & 24 & 2 & 4 & 6 & 8 & 12 & 24 \\
\hline \multirow{3}{*}{ ANKR } & $d$ & 16.75 & 6.68 & 5.75 & 4.31 & 4.37 & 1.60 & 10.10 & 5.62 & 4.38 & 4.24 & 3.00 & 2.86 \\
\hline & $k$ & 6.23 & 3.24 & 3.40 & 2.03 & 2.21 & 1.08 & 4.63 & 2.44 & 2.32 & 1.84 & 1.68 & 0.88 \\
\hline & $y$ & 19.21 & 11.57 & 11.29 & 8.34 & 4.78 & 4.90 & 13.34 & 10.13 & 7.68 & 7.04 & 4.81 & 1.43 \\
\hline \multirow{3}{*}{ CAS1 } & $d$ & 6.90 & 5.45 & 5.71 & 5.00 & 3.43 & 1.30 & 6.57 & 5.79 & 5.67 & 4.94 & 5.12 & 2.02 \\
\hline & $k$ & 6.69 & 4.27 & 4.02 & 2.86 & 2.75 & 1.32 & 6.23 & 4.29 & 4.82 & 2.92 & 3.16 & 1.89 \\
\hline & $y$ & 17.18 & 7.98 & 7.31 & 6.34 & 5.11 & 3.46 & 16.42 & 6.94 & 7.45 & 6.52 & 5.13 & 4.00 \\
\hline \multirow{3}{*}{ ISTA } & $d$ & 19.08 & 7.60 & 5.36 & 3.44 & 2.49 & 1.44 & 8.68 & 6.31 & 4.19 & 3.44 & 3.59 & 1.97 \\
\hline & $k$ & 7.15 & 4.49 & 2.55 & 2.29 & 2.05 & 1.68 & 5.27 & 3.63 & 1.85 & 2.02 & 1.63 & 1.49 \\
\hline & $y$ & 20.85 & 12.04 & 11.68 & 8.02 & 5.26 & 4.71 & 13.71 & 10.79 & 9.07 & 7.02 & 5.83 & 4.68 \\
\hline \multirow{3}{*}{ JCTW } & $d$ & 6.49 & 5.90 & 5.04 & 3.99 & 4.21 & 1.74 & 6.09 & 5.49 & 4.47 & 3.70 & 4.05 & 2.27 \\
\hline & $k$ & 4.18 & 3.67 & 3.45 & 3.18 & 2.44 & 2.28 & 4.39 & 3.34 & 2.90 & 2.87 & 1.90 & 2.15 \\
\hline & $y$ & 17.04 & 14.08 & 15.04 & 13.92 & 11.65 & 6.79 & 16.46 & 11.66 & 12.16 & 11.45 & 9.05 & 6.67 \\
\hline \multirow{3}{*}{ KIR8 } & $d$ & 2.42 & 1.91 & 1.93 & 1.64 & 1.25 & 0.88 & 2.01 & 1.59 & 1.25 & 1.49 & 0.84 & 0.58 \\
\hline & $k$ & 2.74 & 2.31 & 2.18 & 1.87 & 1.46 & 0.89 & 2.23 & 1.77 & 1.79 & 1.21 & 0.96 & 1.15 \\
\hline & $y$ & 10.72 & 7.58 & 6.41 & 5.45 & 4.04 & 2.35 & 8.51 & 5.65 & 5.02 & 4.91 & 3.08 & 3.15 \\
\hline \multirow{3}{*}{ KITG } & $d$ & 3.09 & 2.43 & 2.33 & 1.57 & 2.08 & 1.81 & 3.01 & 2.34 & 2.44 & 2.03 & 2.30 & 0.93 \\
\hline & $k$ & 3.00 & 2.88 & 2.47 & 1.84 & 2.14 & 1.86 & 2.91 & 2.70 & 2.16 & 1.94 & 1.85 & 2.05 \\
\hline & $y$ & 12.98 & 10.99 & 9.20 & 8.12 & 6.00 & 2.86 & 12.03 & 9.79 & 7.63 & 7.91 & 7.55 & 6.95 \\
\hline \multirow{3}{*}{ NKLG } & $d$ & 3.21 & 2.63 & 1.97 & 2.07 & 1.93 & 2.17 & 3.34 & 2.57 & 2.03 & 1.62 & 1.69 & 1.15 \\
\hline & $k$ & 3.69 & 2.16 & 2.17 & 1.88 & 1.83 & 1.02 & 3.48 & 2.58 & 2.33 & 1.54 & 1.45 & 0.20 \\
\hline & $y$ & 11.79 & 10.24 & 6.14 & 5.25 & 7.00 & 3.26 & 11.65 & 9.33 & 7.53 & 6.86 & 5.44 & 4.06 \\
\hline \multirow{3}{*}{ PARK } & $d$ & 3.01 & 3.16 & 2.77 & 2.29 & 2.48 & 1.31 & 2.76 & 2.49 & 2.29 & 1.93 & 1.53 & 0.94 \\
\hline & $k$ & 3.80 & 2.65 & 2.16 & 2.23 & 1.95 & 1.82 & 3.36 & 2.65 & 2.38 & 2.15 & 2.19 & 1.78 \\
\hline & $y$ & 8.01 & 6.62 & 5.44 & 5.10 & 4.64 & 3.36 & 6.57 & 5.38 & 5.04 & 4.52 & 4.23 & 4.04 \\
\hline \multirow{3}{*}{ SALU } & $d$ & 4.13 & 3.54 & 2.31 & 2.55 & 1.46 & 1.46 & 3.86 & 2.96 & 2.32 & 2.37 & 1.27 & 0.99 \\
\hline & $k$ & 4.56 & 4.31 & 4.07 & 3.52 & 2.96 & 0.98 & 3.98 & 3.20 & 3.25 & 2.53 & 2.14 & 0.75 \\
\hline & $y$ & $\begin{array}{l}18.34 \\
\end{array}$ & 12.48 & 10.12 & 8.01 & 7.46 & 3.43 & 16.14 & 10.87 & 10.19 & 8.16 & 5.82 & 4.36 \\
\hline \multirow{3}{*}{ STJ3 } & $d$ & 12.55 & 6.48 & 3.72 & 2.52 & 1.47 & 0.86 & 9.98 & 6.12 & 4.21 & 2.69 & 2.10 & 1.52 \\
\hline & $k$ & 8.29 & 5.96 & 2.45 & 1.56 & 0.94 & 0.49 & 6.10 & 5.09 & 2.64 & 2.24 & 1.48 & 0.93 \\
\hline & $y$ & 14.37 & 11.61 & 5.98 & 4.99 & 2.82 & 1.49 & 11.55 & 10.21 & 6.01 & 5.92 & 3.29 & 1.76 \\
\hline
\end{tabular}

Tablo 3.2: GRE ve GREC senaryolarına ait farklı gözlem süreleri için RMS değerleri (mm)

\begin{tabular}{|c|c|c|c|c|c|c|c|c|c|c|c|c|c|}
\hline \multicolumn{2}{|c|}{ Senaryo } & \multirow{2}{*}{\multicolumn{6}{|c|}{$\begin{array}{c}\text { GRE } \\
\text { Gözlem Süresi (sa) }\end{array}$}} & \multirow{2}{*}{\multicolumn{6}{|c|}{$\begin{array}{c}\text { GREC } \\
\text { Gözlem Süresi (sa) }\end{array}$}} \\
\hline \multirow{2}{*}{ Nokta } & \multirow{2}{*}{ Bileşen } & & & & & & & & & & & & \\
\hline & & 2 & 4 & 6 & 8 & 12 & 24 & 2 & 4 & 6 & 8 & 12 & 24 \\
\hline \multirow{3}{*}{ ANKR } & $d$ & 9.35 & 5.66 & 4.73 & 3.87 & 2.63 & 1.98 & 9.42 & 5.43 & 4.08 & 4.12 & 2.29 & 1.60 \\
\hline & $k$ & 4.61 & 2.32 & 2.35 & 1.59 & 1.79 & 1.06 & 4.50 & 2.23 & 2.29 & 1.70 & 1.80 & 1.26 \\
\hline & $y$ & 12.69 & 9.34 & 8.25 & 5.66 & 4.95 & 3.00 & 12.15 & 9.75 & 7.71 & 5.75 & 4.34 & 3.50 \\
\hline \multirow{3}{*}{ CAS1 } & $d$ & 5.90 & 5.37 & 4.55 & 4.72 & 4.71 & 1.88 & 5.83 & 5.37 & 4.26 & 4.83 & 4.53 & 1.74 \\
\hline & $k$ & 5.71 & 4.18 & 4.35 & 3.15 & 2.80 & 2.10 & 5.51 & 4.04 & 4.38 & 3.27 & 2.95 & 2.63 \\
\hline & $y$ & 13.76 & 7.62 & 7.62 & 6.94 & 5.96 & 3.86 & 14.13 & 8.29 & 7.70 & 7.61 & 6.26 & 2.91 \\
\hline \multirow{3}{*}{ ISTA } & $d$ & 8.57 & 5.36 & 4.26 & 3.09 & 1.48 & 1.92 & 7.70 & 5.21 & 4.15 & 3.13 & 3.43 & 2.12 \\
\hline & $k$ & 5.55 & 3.41 & 1.96 & 1.98 & 1.81 & 1.48 & 5.77 & 3.44 & 1.78 & 2.02 & 1.91 & 1.46 \\
\hline & $y$ & 12.94 & 9.76 & 9.05 & 6.69 & 3.83 & 4.74 & 13.27 & 8.58 & 7.14 & 6.26 & 5.51 & 3.97 \\
\hline \multirow{3}{*}{ JCTW } & $d$ & 6.44 & 5.86 & 4.40 & 3.66 & 3.85 & 2.80 & 6.47 & 5.70 & 4.54 & 4.18 & 3.87 & 2.83 \\
\hline & $k$ & 3.94 & 3.27 & 2.72 & 2.42 & 2.85 & 2.21 & 3.97 & 3.18 & 2.96 & 2.72 & 2.78 & 2.14 \\
\hline & $y$ & 15.61 & 11.41 & 11.47 & 9.32 & 9.35 & 7.16 & 15.93 & 11.73 & 10.66 & 10.24 & 9.37 & 7.44 \\
\hline \multirow{3}{*}{ KIR8 } & $d$ & 1.76 & 1.38 & 1.30 & 1.27 & 0.87 & 0.68 & 1.72 & 1.35 & 1.28 & 1.29 & 0.77 & 0.64 \\
\hline & $k$ & 2.53 & 1.84 & 1.43 & 1.27 & 0.73 & 1.19 & 2.57 & 1.86 & 1.46 & 1.27 & 1.33 & 1.06 \\
\hline & $y$ & 8.22 & 5.90 & 5.04 & 4.60 & 3.83 & 2.65 & 8.74 & 5.76 & 5.13 & 4.65 & 3.36 & 2.47 \\
\hline \multirow{3}{*}{ KITG } & $d$ & 2.81 & 2.19 & 2.01 & 1.99 & 1.87 & 1.54 & 3.19 & 2.57 & 2.50 & 2.08 & 2.24 & 1.09 \\
\hline & $k$ & 3.09 & 2.77 & 2.12 & 2.29 & 1.87 & 1.02 & 3.24 & 2.99 & 2.15 & 1.79 & 2.03 & 1.58 \\
\hline & $y$ & 11.28 & 9.39 & 7.03 & 7.55 & 6.64 & 5.35 & 13.01 & 11.54 & 8.12 & 7.97 & 6.48 & 4.95 \\
\hline \multirow{3}{*}{ NKLG } & $d$ & 3.14 & 2.43 & 1.77 & 1.50 & 1.56 & 0.72 & 3.10 & 2.42 & 1.86 & 1.85 & 1.75 & 1.26 \\
\hline & $k$ & 3.22 & 2.49 & 1.99 & 1.60 & 1.42 & 0.93 & 2.86 & 2.63 & 2.38 & 1.75 & 1.51 & 0.67 \\
\hline & $y$ & 10.58 & 8.26 & 5.58 & 5.22 & 4.96 & 2.49 & 10.58 & 8.54 & 5.39 & 5.39 & 4.23 & 2.51 \\
\hline \multirow{3}{*}{ PARK } & $d$ & 2.93 & 2.77 & 2.23 & 1.73 & 1.50 & 0.73 & 2.83 & 2.45 & 2.06 & 1.78 & 1.43 & 0.76 \\
\hline & $k$ & 3.42 & 3.06 & 2.59 & 2.43 & 2.09 & 1.66 & 3.59 & 2.51 & 2.76 & 2.47 & 2.15 & 1.76 \\
\hline & $y$ & 9.10 & 6.80 & 4.11 & 4.25 & 4.13 & 2.81 & 9.76 & 5.23 & 5.36 & 4.89 & 3.79 & 4.70 \\
\hline \multirow{3}{*}{ SALU } & $d$ & 3.25 & 2.86 & 2.17 & 2.24 & 1.24 & 0.96 & 3.08 & 2.66 & 2.11 & 1.50 & 1.54 & 1.09 \\
\hline & $k$ & 3.89 & 3.21 & 3.06 & 2.56 & 1.95 & 0.86 & 4.27 & 3.17 & 2.95 & 2.36 & 1.90 & 0.83 \\
\hline & $y$ & 16.07 & 12.72 & 10.34 & 8.22 & 6.44 & 5.50 & 18.42 & 13.62 & 11.32 & 10.50 & 8.02 & 5.65 \\
\hline \multirow{3}{*}{ STJ3 } & $d$ & 9.63 & 5.99 & 4.22 & 2.95 & 2.19 & 1.35 & 9.61 & 5.33 & 3.91 & 1.97 & 2.07 & 1.20 \\
\hline & $k$ & 5.25 & 3.74 & 2.40 & 2.05 & 1.35 & 0.77 & 6.23 & 4.58 & 2.02 & 1.55 & 1.24 & 0.77 \\
\hline & $y$ & 11.34 & 8.04 & 5.63 & 5.10 & 3.77 & 2.33 & 11.11 & 8.81 & 5.17 & 5.11 & 3.56 & 2.40 \\
\hline
\end{tabular}

Trimble RTX servisi ile elde edilen sonuçlar incelendiğinde; yatay bileşende, ANKR, ISTA ve STJ3 noktaları hariç tüm 
noktalarda bütün senaryolarla 2 saatlik gözlemlerle bile $\mathrm{mm}$ mertebesinde doğruluk değerlerinin elde edilebildiği görülmektedir. ANKR, ISTA ve STJ3 noktalarında ise farklı uydu kombinasyonu çözümleri ile 2 saatlik gözlem süresinde mm mertebesinde doğruluğa ulaşılabilmiştir. Düşey bileşende ise; sadece GPS çözümlerinde mm mertebesinde doğruluk çoğunlukla 6-8 saatlik gözlemler ile sağlanabilmiştir. Ancak, bu doğruluk değeri GR senaryosunda 4-6; GRE ve GREC senaryolarında 4 saatlik gözlem süreleriyle elde edilmiştir.

Çalışmada ayrıca, GNSS gözlem süresi ve nokta enlemlerinin Çoklu-GNSS çözümlerine olan etkisini incelemek amacıyla Eşitlik 6'daki doğruluk fonksiyonları belirlenmiştir (Eckl vd., 2001; Saraçoğlu ve Şanl1, 2020; Şanl1 \& Tekic, 2010).

$S_{i}(\varphi, T)=\sqrt{\frac{a_{i}}{T}+\frac{b_{i} \varphi^{2}}{T}+c_{i}+d_{i} \varphi^{2}}$

Burada; $i$ indisi koordinat bileşenini $(k, d, y), S$ ilgili koordinat bileşenine ait RMS değerini, $a_{i}, b_{i}, c_{i}$ ve $d_{i}$ fonksiyon parametrelerini, $T$ saat biriminde gözlem süresini ve $\varphi$ derece biriminde enlem bilgisini ifade etmektedir. Bu eşitlikte $a_{i}$ parametresi sadece gözlem süresine, $b_{i}$ parametresi hem gözlem süresine hem de istasyon enlem bilgisine ve $d_{i}$ parametresi istasyon enlem bilgisine göre değişmektedir. $c_{i}$ parametresi ise gözlem süresi ve enlem bilgisi dışındaki olası değişkenler ile ilişkilendirilmiştir.

Her bir senaryoya ait fonksiyon parametreleri En Küçük Kareler (EKK) yöntemiyle belirlenmiş ve Tablo 4'te gösterilmiştir (Koch, 2013). Ayrıca bu parametrelerin anlamlılığı Student T-testi ile incelenmiştir. Tablo 4 incelendiğinde tüm fonksiyonlarda sadece gözlem süresine bağlı parametrenin ( $a_{i}$ parametresinin) anlamlı; yani test değerinin sınır değerden büyük olduğu (2.003), diğer parametrelerin ise anlamsız olduğu görülmektedir.

Tablo 4: Doğruluk fonksiyonlarının parametre değerleri

\begin{tabular}{|c|c|c|c|c|c|c|c|c|c|c|c|c|}
\hline & \multicolumn{3}{|c|}{ G } & \multicolumn{3}{|c|}{ GR } & \multicolumn{3}{|c|}{ GRE } & \multicolumn{3}{|c|}{ GREC } \\
\hline Parametre & Değer & $\begin{array}{l}\text { Standart } \\
\text { Sapmas1 }\end{array}$ & $\begin{array}{c}\text { Test } \\
\text { Değeri }\end{array}$ & Değer & $\begin{array}{l}\text { Standart } \\
\text { Sapması }\end{array}$ & $\begin{array}{c}\text { Test } \\
\text { Değeri }\end{array}$ & Değer & $\begin{array}{l}\text { Standart } \\
\text { Sapması }\end{array}$ & $\begin{array}{c}\text { Test } \\
\text { Değeri }\end{array}$ & Değer & $\begin{array}{l}\text { Standart } \\
\text { Sapması }\end{array}$ & $\begin{array}{c}\text { Test } \\
\text { Değeri }\end{array}$ \\
\hline$a_{D}\left(\mathrm{~mm}^{2} . \mathrm{sa}\right)$ & 203.572 & 72.122 & 2.823 & 76.071 & 23.660 & 3.215 & 73.057 & 21.630 & 3.378 & 67.690 & 20.755 & 3.261 \\
\hline $\begin{array}{c}b_{D}\left(\mathrm{~mm}^{2} \cdot \mathrm{sa}\right. \\
\left./ \mathrm{der}^{2}\right)\end{array}$ & -0.001 & 0.031 & 0.018 & 0.003 & 0.010 & 0.257 & 0.001 & 0.009 & 0.132 & 0.002 & 0.009 & 0.219 \\
\hline$c_{D}\left(\mathrm{~mm}^{2}\right)$ & -14.945 & 17.778 & 0.841 & -2.204 & 5.832 & 0.378 & -2.240 & 5.332 & 0.420 & -1.430 & 5.116 & 0.280 \\
\hline$d_{D}\left(1 / \mathrm{der}^{2}\right)$ & 0.001 & 0.008 & 0.098 & 0.001 & 0.002 & 0.472 & 0.001 & 0.002 & 0.417 & 0.001 & 0.002 & 0.311 \\
\hline$a_{K}\left(\mathrm{~mm}^{2} . \mathrm{sa}\right)$ & 45.807 & 12.662 & 3.618 & 31.482 & 7.994 & 3.938 & 29.237 & 6.293 & 4.646 & 33.366 & 7.393 & 4.513 \\
\hline $\begin{array}{c}b_{K}\left(\mathrm{~mm}^{2} . \mathrm{sa}\right. \\
\left./ \mathrm{der}^{2}\right)\end{array}$ & 0.007 & 0.005 & 1.292 & 0.004 & 0.003 & 1.180 & 0.003 & 0.003 & 1.155 & 0.002 & 0.003 & 0.732 \\
\hline$c_{K}\left(\mathrm{~mm}^{2}\right)$ & 1.082 & 3.121 & 0.347 & 0.582 & 1.970 & 0.296 & 0.813 & 1.551 & 0.524 & 0.313 & 1.822 & 0.172 \\
\hline$d_{K}\left(1 / \operatorname{der}^{2}\right)$ & -0.001 & 0.001 & 0.727 & 0.000 & 0.001 & 0.018 & 0.000 & 0.001 & 0.051 & 0.000 & 0.001 & 0.224 \\
\hline$a_{Y}\left(\mathrm{~mm}^{2} . \mathrm{sa}\right)$ & 496.579 & 83.784 & 5.927 & 329.729 & 54.622 & 6.037 & 339.709 & 45.701 & 7.433 & 386.198 & 54.714 & 7.058 \\
\hline $\begin{array}{c}b_{Y}\left(\mathrm{~mm}^{2} . \mathrm{sa}\right. \\
\left./ \mathrm{der}^{2}\right)\end{array}$ & -0.006 & 0.035 & 0.164 & -0.003 & 0.023 & 0.145 & -0.024 & 0.019 & 1.259 & -0.033 & 0.023 & 1.414 \\
\hline$c_{Y}\left(\mathrm{~mm}^{2}\right)$ & 12.871 & 20.653 & 0.623 & 20.200 & 13.464 & 1.500 & 12.610 & 11.265 & 1.119 & 13.190 & 13.487 & 0.978 \\
\hline$d_{Y}\left(1 / \operatorname{der}^{2}\right)$ & -0.008 & 0.009 & 0.865 & -0.007 & 0.006 & 1.152 & -0.003 & 0.005 & 0.565 & -0.003 & 0.006 & 0.598 \\
\hline
\end{tabular}

Fonksiyonda sadece gözlem süresine bağlı parametrenin anlamlı olarak ortaya çıktığı belirlendikten sonra fonksiyon Eşitlik 7'deki şekliyle yeniden düzenlenmiştir. Her bir koordinat bileşeni için k katsayı değerleri EKK yöntemi kullanılarak hesaplanmış ve sonuçlar Tablo 5'te sunulmuştur.

$S_{i}(T)=\frac{k_{i}}{\sqrt{T}}$ 
Burada $k_{i}$, fonksiyon parametresini ifade etmektedir.

Tablo 5: Senaryolara ait gözlem süresine bağı parametre değerleri

\begin{tabular}{cccc}
\hline & $\boldsymbol{k}_{\boldsymbol{D}}$ & $\boldsymbol{k}_{\boldsymbol{K}}$ & $\boldsymbol{k}_{\boldsymbol{Y}}$ \\
\hline $\mathbf{G}$ & 12.61 & 7.51 & 21.97 \\
\hline $\mathbf{G R}$ & 8.97 & 6.38 & 18.70 \\
\hline GRE & 8.58 & 6.11 & 17.88 \\
\hline GREC & 8.40 & 6.29 & 18.67 \\
\hline
\end{tabular}

Tablo 5 incelendiğinde en küçük fonksiyon parametresi değerinin doğu bileşeni için GREC ile; kuzey ve yukarı bileşenleri için ise GRE senaryosu ile elde edildiği görülebilmektedir. Bu sonuca dayanarak da uydu kombinasyonları ile elde edilen çözümlerin daha yüksek doğruluğa sahip olduğu söylenebilir.

\section{Sonuçlar}

$\mathrm{Bu}$ çalışmada internet tabanlı bir servis olan Trimble RTX ile gerçekleştirilen Çoklu-GNSS çözümlerinin doğruluğu araştırılmıştır. Seçilen 10 adet noktanın 10’ar günlük verileri 6 farklı gözlem süresinde değerlendirilmiştir. RMS değerleri incelendiğinde doğruluğun gözlem süresine bağlı olarak değiştiği görülmektedir. Analizlerde en uzun süre olarak 24 saat seçilmiştir. Bu gözlem süresine ilişkin sonuçlarda tüm sistemlerin en yüksek doğruluğa sahip olduğu görülmektedir. Analizlerde kullanılan ürünlerin doğrulukları ve Trimble RTX servisinin analizlerde standart bir çözüm kullandığı düşünüldüğünde elde edilebilecek maksimum doğruluğa 24 saatlik gözlemlerde ulaşılabilmektedir. Çalışmada kullanılan farklı uydu kombinasyonlarının 24 saatlik gözlem verisi ile elde edilen doğruluk değerlerinin, sadece GPS verisi kullanılarak elde edilen doğruluğa yakınsadığı görülmüştür. Ancak, bu durum farklı uydu kombinasyonlarının çözümlere herhangi bir katkısı olmadığı anlamına gelmemektedir. Gözlem süresi kısaldıkça bu etki ortaya çıkmaktadır. Sadece GPS çözümü ile 6-8 saatlik gözlem süresinde elde edilen doğruluğa GRE kombinasyonu ile 4 saatte ulaşılabildiği gösterilmiştir. Ayrıca, ÇokluGNSS çözüm doğruluklarının gözlem süresi ve enleme bağlı olup olmadığını incelemek için doğruluk fonksiyonu belirlenmiştir. Fonksiyonda $a_{i}$ parametresi sadece gözlem süresine, $b_{i}$ parametresi hem gözlem süresine hem de istasyon enlem bilgisine ve $d_{i}$ parametresi istasyon enlem bilgisine bağlılı̆̆ ifade etmektedir. $c_{i}$ parametresi ise gözlem süresi ve enlem bilgisi dışındaki olası değişkenler ile olan ilişkiyi ortaya koymaktadır. Tüm senaryolarda ve koordinat bileşenlerinde, doğruluk fonksiyonunun sadece gözlem süresine bağlı parametreleri ( $a_{i}$ parametresi) anlamlı çıkmış diğer enlem ve olası tüm değişkenlere ait parametreler $\left(b_{i} c_{i}\right.$ ve $d_{i}$ parametreleri) anlamsız çıkmıştır. Eğer sadece $d_{i}$ parametresi anlamlı çıksaydı bu durum çözümlerden elde edilen doğruluğun sadece enleme bağlı olduğu; sadece $a_{i}, b_{i}$ ve $d_{i}$ parametreleri anlamlı olsayd bu durumda ise çözümlerin doğruluğunun hem noktanın enlemine hem de gözlem süresine bağlı olduğu ortaya çıkacaktı. Sadece $a_{i}$ parametresinin anlamlı çıkması, Çoklu-GNSS çözümlerinin enleme veya ek bir parametreye bağlı olmadığını, sadece gözlem süresine bağlı olduğunu göstermektedir. Tüm sonuçlar incelendiğinde Galileo ve BeiDou sistemlerinin GPS ve GLONASS sistemlerine konum doğruluğu açısından katkı sağladığı söylenebilir. Özellikle kısa süreli gözlemlerde sadece GPS ölçülerini kullanmak yerine doğruluğu arttırmak için farklı uydu kombinasyonları tercih edilebilir. Bununla birlikte Trimble RTX servisi ile mm mertebesinde doğruluk elde edilebildiğinden; yüksek doğruluk gerektiren mühendislik ölçmeleri çalışmalarında bu servis kullanılabilir.

\section{Teşekkür}

$\mathrm{Bu}$ çalışmada IGS-MGEX ağına ait noktalar kullanılmıştır. Verileri sağladığı için IGS kurumuna ve verilerin değerlendirildiği Trimble RTX servisini geliştiren araştırmacılara teşekkür ederiz. Şekil 1 Generic Mapping Tools (GMT) 
kullanılarak çizilmiştir (Wessel \& Smith, 1998).

\section{Yazar Katkısı}

Gizem Sezer: Tasarım, Literatür taraması, Analiz ve yorumlama, Yazım, Veri toplama ve işleme. Ali Hasan Doğan: Denetleme, Yazım, Literatür taraması, Analiz ve yorumlama, Veri toplama ve işleme. Bahattin Erdoğan: Fikir, Tasarım, Analiz ve yorumlama, Denetleme, Yazım.

\section{Kaynaklar}

Albayrak, M., Erdoğan, B., \& Erkaya, H. (2020). Performance analysis of web-based relative and precise point positioning techniques with different satellite visibility conditions. Boletim de Ciências Geodésicas, 26(1).

Bisnath, S., \& Gao, Y. (2009). Current state of precise point positioning and future prospects and limitations. Observing our changing earth, s. 615-623. Springer, Berlin, Heidelberg.

Doucet, K., Herwig, M., Kipka, A., Kreikenbohm, P., Landau, H., Leandro, R., Moessmer, M., \& Pagels, C. (2012). Introducing ambiguity resolution in webhosted global multi-GNSS precise positioning with Trimble RTX-PP. Proceedings of the 25th International Technical Meeting of The Satellite Division of the Institute of Navigation, 17, 1115-1125.

Eckl, M. C., Snay, R. A., Soler, T., Cline, M. W., \& Mader, G. L. (2001). Accuracy of GPS-derived relative positions as a function of interstation distance and observing-session duration. Journal of geodesy, 75(12), 633-640.

Erdoğan, B., Kayacık, O., \& Doğan, A.H. (2019). Hassas mutlak nokta konumlamada GIPSY-OASIS II v6.4 yazılımı ile elde edilen varyans kovaryans matrisinin güvenirliğinin araştırılması. Jeodezi ve Jeoinformasyon Dergisi , 6(2) , 75-86 .

Erdoğan, B., \& Doğan, A.H. (2019). Scaling of the variance covariance matrix obtained from Bernese software. Acta Geodaetica et Geophysica, 54(2), 197-211.

Gao, Y., \& Shen, X. (2001). Improving ambiguity convergence in carrier phase-based precise point positioning. Proceedings of the 14th international technical meeting of the Satellite Division of the Institute of Navigation, 1532-1539.

Geng, J., Teferle, F. N., Meng, X., \& Dodson, A. H. (2011). Towards PPP-RTK: Ambiguity resolution in real-time precise point positioning. Advances in space research, 47(10), 1664-1673.

Hampel, F. R., Ronchetti, E. M., Rousseeuw, P. J., \& Stahel, W. A. (2011). Robust statistics: the approach based on influence functions. John Wiley \& Sons.

Hekimoğlu, Ş. (2005). Do Robust Methods Identify Outliniers More Reliably Than Conventional Tests for Outliniers ?. ZfV magazine for geodesy, geoinformation and land management, 3, 174-180.

Koch, K.-R. (2013). Parameter estimation and hypothesis testing in linear models, Springer Science \& Business Media.

Kouba, J., \& Héroux, P. (2001). Precise point positioning using IGS orbit and clock products. GPS solutions, 5(2), 12-28.

Li, X., Ge, M., Dai, X., Ren, X., Fritsche, M., Wickert, J., \& Schuh, H. (2015). Accuracy and reliability of multi-GNSS real-time precise positioning: GPS, GLONASS, BeiDou, and Galileo. Journal of Geodesy, 89(6), 607-635.

Montenbruck, O., Steigenberger, P., Prange, L., Deng, Z., Zhao, Q., Perosanz, F., Romero, I., Noll, C., Stürze, A., \& Weber, G. (2017). The Multi-GNSS Experiment (MGEX) of the International GNSS Service (IGS)-achievements, prospects and challenges. Advances in Space Research, 59(7), 1671-1697.

Öcalan, T., Erdoğan, B., \& Tunalığlu, N. (2013). Analysis of web-based online services for GPS relative and precise point positioning techniques. Boletim de ciencias geodesicas, 19(2), 191-207.

Öcalan T. (2015). GNSS Ağlarında GPS Hassas Nokta Konumlama (GPS-PPP) Tekniği Yaklaşımlı Çözümler (Doktora Tezi). Yıldız Teknik Üniversitesi, Fen Bilimleri Enstitüsü, İstanbul, Türkiye.

Öğ̈̈tcü, S. (2019). The contribution of Multi-GNSS Experiment (MGEX) to precise point positioning over Turkey: Consideration of observation time and satellite geometry. El-Cezeri Journal of Science and Engineering, 6(3), 642-658.

Öğ̈ütcü, S. (2020). Assessing the contribution of Galileo to GPS+ GLONASS PPP: Towards full operational capability. Measurement, 151, 
107143.

Rizos, C., Janssen, V., Roberts, C., \& Grinter, T. (2012), Precise Point Positioning: Is the Era of Differential GNSS Positioning Drawing to an End? FIG Working Week 2012, Roma, İtalya.

Saraçoğlu, A., \& Şanli, D. U. (2020). Effect of meteorological seasons on the accuracy of GPS positioning. Measurement, $152,107301$.

Soler, T., Michalak, P., Weston, N. D., Snay, R. A., \& Foote, R. H. (2006). Accuracy of OPUS solutions for 1-to 4-h observing sessions. GPS solutions, 10(1), 45-55.

Soycan, M., \& Öcalan, T. (2011). A regression study on relative GPS accuracy for different variables. Survey Review, 43(320), $137-149$.

Şanl1, D. U., \& Engin, C. (2009). Accuracy of GPS positioning over regional scales. Survey Review, 41(312), 192-200.

Şanlı, D. U., \& Tekiç, S. (2010). Accuracy of GPS precise point positioning: A tool for GPS accuracy prediction. Lambert Acad. Publ.

Toluc, A. B. (2016). Multi-GNSS Precise Point Positioning Using GPS, GLONASS and Galileo (Yüksek Lisans Tezi). The Ohio State University, Graduate School of The Ohio State University, Ohio, Amerika Birleşik Devletleri.

Tut, İ., Şanlı, D. U., Erdoğan, B., \& Hekimoğlu, Ş. (2013). Efficiency of BERNESE single baseline rapid static positioning solutions with search strategy. Survey review, 45(331), 296-304.

Wang, J., Wu, Z., Semmling, M., Zus, F., Gerland, S., Ramatschi, M., Ge, M., Wickert, J., \& Schuh, H. (2019). Retrieving Precipitable Water Vapor From Shipborne Multi-GNSS Observations. Geophysical Research Letters, 46(9), 5000-5008.

Wessel, P., \& Smith, W. H. (1998). New, improved version of Generic Mapping Tools released. Eos, Transactions American Geophysical Union, 79(47), 579-579.

Xia, F., Ye, S., Xia, P., Zhao, L., Jiang, N., Chen, D., \& Hu, G. (2019). Assessing the latest performance of Galileo-only PPP and the contribution of Galileo to Multi-GNSS PPP. Advances in space research, 63(9), 2784-2795.

Yapıc1, T. (2007). Influences of interplanetary magnetic field on the variability of aerospace media (Yüksek Lisans Tezi), Orta Doğu Teknik Üniversitesi, Fen Bilimleri Enstitüsü, Ankara, Türkiye.

Zumberge, J. F., Heflin, M. B., Jefferson, D. C., Watkins, M. M., \& Webb, F. H. (1997). Precise point positioning for the efficient and robust analysis of GPS data from large networks. Journal of geophysical research: solid earth, 102(B3), 5005-5017.

URL-1: International GNSS Service, http://mgex.igs.org/, (Erişim Tarihi:1 Nisan 2020).

URL-2: Web Based Trimble RTX Service, https://www.trimblertx.com/UploadForm.aspx, (Erişim Tarihi:1 Nisan 2020).

URL-3: Space Weather Archive, https://www.spaceweatherlive.com/en/archive, (Erişim Tarihi:3 Nisan 2020). 